\title{
Cloud algorithm-driven oximetry-based diagnosis of obstructive sleep apnoea in symptomatic habitually snoring children
}

\author{
Zhifei $\mathrm{Xu}^{1}$, Gonzalo C. Gutiérrez-Tobal ${ }^{2}$, Yunxiao $\mathrm{Wu}^{3}$, Leila Kheirandish-Gozal ${ }^{4}$, \\ Xin $\mathrm{Ni}^{3,5}$, Roberto Hornero ${ }^{2}$ and David Gozal ${ }^{4,5}$ \\ Affiliations: ${ }^{1}$ Respiratory Dept, Beijing Children's Hospital, Capital Medical University, National Center for \\ Children's Health, Beijing, People's Republic of China. ${ }^{2}$ Biomedical Engineering Group, Universidad de \\ Valladolid, Valladolid, Spain. ${ }^{3}$ Otolaryngology, Head and Neck Surgery Dept, Beijing Children's Hospital, \\ Capital Medical University, National Center for Children's Health, Beijing, People's Republic of China. ${ }^{4}$ Dept of \\ Child Health, University of Missouri School of Medicine, Columbia, MO, USA. ${ }^{5}$ David Gozal and Xin Ni \\ contributed equally to this article as lead authors and supervised the work.
}

\section{Correspondence:}

David Gozal, Dept of Child Health, University of Missouri School of Medicine, 400 N. Keene Street, Suite 010 , Columbia, MO 65201, USA.

E-mail: gozaldahealth.missouri.edu

@ERSpublications

The scarcity of sleep studies reduces access and restricts objective diagnosis of obstructive sleep apnoea in children. Overnight oximetry linked via Bluetooth to a smartphone and cloud algorithm provides scalable diagnostic capability. http://ow.ly/OCx230mNE4n

Cite this article as: $\mathrm{Xu} \mathrm{Z,} \mathrm{Gutiérrez-Tobal} \mathrm{GC,} \mathrm{Wu} \mathrm{Y,} \mathrm{et} \mathrm{al.} \mathrm{Cloud} \mathrm{algorithm-driven} \mathrm{oximetry-based}$ diagnosis of obstructive sleep apnoea in symptomatic habitually snoring children. Eur Respir J 2019; 53: 1801788 [https://doi.org/10.1183/13993003.01788-2018].

ABSTRACT The ability of a cloud-driven Bluetooth oximetry-based algorithm to diagnose obstructive sleep apnoea syndrome (OSAS) was examined in habitually snoring children concurrently undergoing overnight polysomnography.

Children clinically referred for overnight in-laboratory polysomnographic evaluation for suspected OSAS were simultaneously hooked to a Bluetooth oximeter linked to a smartphone. Polysomnography findings were scored and the apnoea/hypopnoea index (AHIPSG) was tabulated, while oximetry data yielded an estimated AHIOXI using a validated algorithm.

The accuracy of the oximeter in identifying correctly patients with OSAS in general, or with mild (AHI 1-5 events $\left.\cdot \mathrm{h}^{-1}\right)$, moderate $\left(5-10\right.$ events $\left.\cdot \mathrm{h}^{-1}\right)$ or severe $\left(>10\right.$ events $\left.\cdot \mathrm{h}^{-1}\right)$ OSAS was examined in 432 subjects (6.5 \pm 3.2 years), with 343 having AHIPSG $>1$ event $\cdot \mathrm{h}^{-1}$. The accuracies of AHIoxI were consistently $>79 \%$ for all levels of OSAS severity, and specificity was particularly favourable for AHI $>10$ events $\cdot \mathrm{h}^{-1}$ (92.7\%). Using the criterion of AHIPSG $>1$ event $\cdot \mathrm{h}^{-1}$, only $4.7 \%$ of false-negative cases emerged, from which only $0.6 \%$ of cases showed moderate or severe OSAS.

Overnight oximetry processed via Bluetooth technology by a cloud-based machine learning-derived algorithm can reliably diagnose OSAS in children with clinical symptoms suggestive of the disease. This approach provides virtually limitless scalability and should alleviate the substantial difficulties in accessing paediatric sleep laboratories while markedly reducing the costs of OSAS diagnosis.

Published in volume 53, issue 2 of the European Respiratory Journal on 21 Feb 2019; republished 7 May 2021 with amendments to the author list footnote indicating equal contribution of the lead authors. 


\section{Introduction}

Obstructive sleep apnoea/hypopnoea syndrome (OSAS) has emerged in recent decades as a highly prevalent disease in children all over the world, and is estimated to affect $2-5 \%$ of all children. However, the cardinal symptom of OSAS in children is habitual snoring, which affects a much higher proportion of children (range 6-25\%). Furthermore, paediatric OSAS has been consistently associated with increased risk of major end-organ adverse consequences affecting neurocognitive, behavioural, cardiovascular and metabolic systems, ultimately resulting in overall declines in health and quality of life, as well as increased healthcare costs [1-4]. Based on current guidelines, nocturnal polysomnography (PSG) in an accredited sleep laboratory is considered the gold-standard approach to diagnose OSAS in children $[1,5,6]$. However, the scarcity of paediatric sleep laboratories around the world, the elevated costs of PSG and their labour-intensive nonscalable characteristics, and the obvious inconvenience to parents and children have led to the unfortunate reality that only a minute proportion of symptomatic habitually snoring children are evaluated objectively before undergoing adenotonsillectomy, the first line of therapy $[7,8]$.

In an effort to overcome these problems and expand the accessibility and objectivity of OSAS diagnosis, many alternative methodologies have been developed, ranging from questionnaires to simplified multichannel studies, and even exploration of diagnostic biomarker panels [9-13]. Nocturnal oximetry was proposed initially as a screening tool for OSAS in symptomatic children [11, 13], and this approach has gained increasing popularity despite exhibiting favourable specificity yet limited sensitivity, while also being marred by interscorer reliability issues, particularly at the low end of OSAS severity, as well as scalability concerns [11]. To overcome these issues, several investigative groups including ours have proposed a variety of automated procedures that circumvent the subjectivity of oximetry recording interpretation [14-28]. In this context, we have reported on the application of machine-learning procedures in the analysis of nocturnal oximetry recordings among children referred for clinical evaluation of suspected OSAS, and the derivation and validation of a diagnostic algorithm in a very large cohort of $>4000$ children [29]. Here, we furthered our quest for a scorer-independent scalable diagnostic approach of paediatric OSAS by exploring and comparing the diagnostic performance of a Bluetooth-enabled oximeter coupled to a smartphone for data transmission and derivation of the estimated apnoea/hypopnoea using a cloud-based algorithm when tested concurrent with a PSG study in the laboratory.

\section{Patients and methods}

Subjects

Consecutive, otherwise healthy, habitually snoring symptomatic children ( $\geqslant 3$ nights per week) aged 2-15 years who were referred for suspected OSAS were recruited from Beijing Children's Hospital, Capital Medical University (Beijing, China) between June 1, 2017 and June 1, 2018. All participants underwent an overnight PSG evaluation while concurrently wearing a commercially and readily available Bluetooth oximeter linked to an Android smartphone via a custom designed application (Serenium, Palo Alto, CA, USA). Written informed consent and assent were obtained from parents and children, respectively (for children aged $>7$ years). The study was approved by the ethics committee of Beijing Children's Hospital affiliated to Capital Medical University (protocol \#2017-151), and received approval for processing of the de-identified oximetry recordings from the University of Chicago human subject committee (protocol \#IRB14-1241).

\section{Exclusion criteria}

Children who were known to be suffering from congenital heart disease, systemic or pulmonary hypertension, diabetes mellitus or dyslipidaemia, those with craniofacial anomalies, neuromuscular disease or defined genetic syndromes were excluded. In addition, children with any known acute or chronic illness, or who received previous treatment for OSAS were excluded. Children with recordings from either PSG or Bluetooth oximeter lasting $<3 \mathrm{~h}$ were excluded.

\section{Anthropometry}

All children were weighed on a calibrated scale and their weights were recorded to the nearest $0.1 \mathrm{~kg}$. Height (to $0.1 \mathrm{~cm}$ ) was measured using a stadiometer. The body mass index (BMI) and BMI $\mathrm{z}$-score were calculated using Chinese normative datasets [30]. The definition of obesity in our study was BMI z-score $\geqslant 1.65$.

\section{Polysomnography}

Children were monitored during the PSG using a digital acquisition system (Compumedics E; Compumedics, Melbourne, Australia or ALICE 5; Philips Respironics, Amsterdam, the Netherlands). No coffee, tea, cola-containing products or sedative hypnotics were taken before sleep. Total sleep time was $>7.5 \mathrm{~h}$. PSG monitoring included the following parameters: electroencephalogram from four leads (C3/A2, C4/A1, O1/A2, O2/A3), bilateral electro-oculogram, electromyogram of mentalis activity and bilateral anterior tibialis, chest and abdominal movements, ECG, arterial oxyhaemoglobin saturation and plethysmographic signal by pulse 
oximetry, air flow thermistor and nasal pressure cannula, snoring sensor and body position. Sleep data were scored manually by experienced paediatric PSG technicians according to the scoring manual published by the American Academy of Sleep Medicine (AASM) [31]. Oxygen desaturation index (ODI3\%) was defined as the number of $\geqslant 3 \%$ arterial oxygen desaturations per hour of sleep. The definition of arousal was based on the AASM guidelines. The diagnosis of children with OSAS was defined by the presence of an obstructive apnoea/hypopnoea index (AHIPSG) $\geqslant 1$ event.h ${ }^{-1}$ of total sleep time according to the most frequent clinical practice, as described in the 2012 American Academy of Pediatrics consensus guideline for the diagnosis and management of childhood OSAS [1]. Primary snoring was defined as AHI $<1$ event $\cdot \mathrm{h}^{-1}$. Mild OSAS was defined as AHIPSG $\geqslant 1$ event $\cdot \mathrm{h}^{-1}$ and AHIPSG $<5$ events $\cdot \mathrm{h}^{-1}$, moderate OSAS was defined as AHIPSG $\geqslant 5$ events $\cdot h^{-1}$ and severe OSAS was defined as AHIPSG $\geqslant 10$ events $\cdot h^{-1}$.

\section{Oximetry data processing}

Oximetry signals from the Bluetooth oximeter were transferred via the smartphone to the cloud and were all rounded to the second decimal place. Artefacts were then automatically removed according to the method proposed by MAGALANG et al. [32]. Signals were automatically processed using the algorithm previously developed and validated [29], which consisted of a multilayer perceptron (MLP) model with the ability to estimate AHI automatically. MLP constitutes an artificial neural network that is typically arranged in three layers of mathematical units called neurons: input, hidden and output [29], and Matlab R2016b (MathWorks, Cambridge, UK) was used to implement feature extraction and classification stages. Accordingly, an estimate of the AHI was computed (AHIOxI), and compared with AHIPSG.

\section{Statistical analyses}

SPSS Statistics software (version 20; IBM, Chicago, IL, USA) was used, and data are presented as mean \pm SD. Intraclass correlation coefficient (ICC) was used to directly assess the agreement between the AHIPSG and AHIOXI, as well as Bland-Altman and Cohen's $\kappa$ [33]. In addition, the diagnostic performance for three cut-offs (AHI 1 event $\cdot \mathrm{h}^{-1}, 5$ events $\cdot \mathrm{h}^{-1}$ and 10 events $\cdot \mathrm{h}^{-1}$ ) was assessed by means of sensitivity, specificity, positive likelihood ratio $\left(\mathrm{LR}^{+}\right)$, negative likelihood ratio $\left(\mathrm{LR}^{-}\right)$and accuracy. Plotting of the LR values was performed using a freely available web-based calculator developed by Alan Schwartz (http://araw.mede.uic. edu/cgi-bin/testcalc.pl? $\mathrm{DT}=\& \mathrm{Dt}=\& \mathrm{dT}=\& \mathrm{dt}=\& 2 \times 2=$ Compute). For comparisons of continuous variables across clinical groupings, Kruskal-Wallis (nonparametric) or Mann-Whitney tests were used as appropriate. For comparisons of discrete variables, a Chi-squared test was used. A p-value $<0.05$ was considered to be indicative of statistical significance.

\section{Results}

432 children completed the study out of 435 who were approached and agreed to participate. The reasons for inability to complete the study was related to intolerance of the PSG equipment by the three very young children (ages 2 years, 2.5 years and 3 years), which led to them spending most of the recording time awake or without an appropriate PSG recording montage. Table 1 provides the demographic and anthropometric characteristics of the cohort as well as their PSG findings.

TABLE 1 Demographic, anthropometric and polysomnographic characteristics of 432 symptomatic habitually snoring Chinese children undergoing overnight polysomnography and concurrent Bluetooth oximetry for suspected obstructive sleep apnoea syndrome (OSAS)

\begin{tabular}{|c|c|c|c|c|}
\hline & All participants & $\begin{array}{c}\text { Primary snoring } \\
\text { AHIPSG } \leqslant 1 \text { event } \cdot h^{-1}\end{array}$ & $\begin{array}{l}\text { OSAS AHIPSG } \\
>1 \text { event } \cdot h^{-1}\end{array}$ & $\begin{array}{l}\text { OSAS AHIPSG } \\
>5 \text { events } \cdot h^{-1}\end{array}$ \\
\hline Subjects & 432 & 89 & 343 & 171 \\
\hline Male \% & 65.3 & 62.8 & 69.4 & $64.5 \%$ \\
\hline BMI (\% obese) & $17.8 \pm 4.5(26.3)$ & $16.6 \pm 3.9(23.2)$ & $18.3 \pm 4.7(26.7)$ & $19.2 \pm 5.4(33.1)^{* *}$ \\
\hline Total sleep time min & $474.1 \pm 54.4$ & $460.4 \pm 72.2$ & $478.1 \pm 47.8$ & $471.7 \pm 48.4$ \\
\hline ODl $3 \%$ events $\cdot h^{-1}$ & $6.7 \pm 16.2$ & $0.2 \pm 0.7$ & $8.3 \pm 17.7$ & $14.8 \pm 21.4^{\#}$ \\
\hline $\mathrm{SpO}_{2}$ nadir & $89.8 \pm 7.2$ & $94.3 \pm 2.0$ & $88.6 \pm 7.6$ & $85.2 \pm 9.0^{\#}$ \\
\hline \multicolumn{5}{|c|}{$\begin{array}{l}\text { Data are presented as } \mathrm{n} \text { or mean } \pm \mathrm{SD} \text {, unless otherwise stated. AHIPSG: apnoea/hypopnoea index measured using polysomnography; } \mathrm{BMI} \text { : body } \\
\text { mass index; } \mathrm{AHI} \text { : apnoea/hypopnoea index; IQR: interquartile range; ODI } 3 \% \text { : oxygen desaturation index (the number of } \geqslant 3 \% \text { arterial oxygen } \\
\text { desaturations per hour of sleep); } \mathrm{SpO}_{2} \text { : arterial oxygen saturation measured by pulse oximetry. }{ }^{* *}: \mathrm{p}<0.01 \text { versus all others; }{ }^{\#} \text { : p<0.0001 versus } \\
\text { primary snoring. }\end{array}$} \\
\hline
\end{tabular}




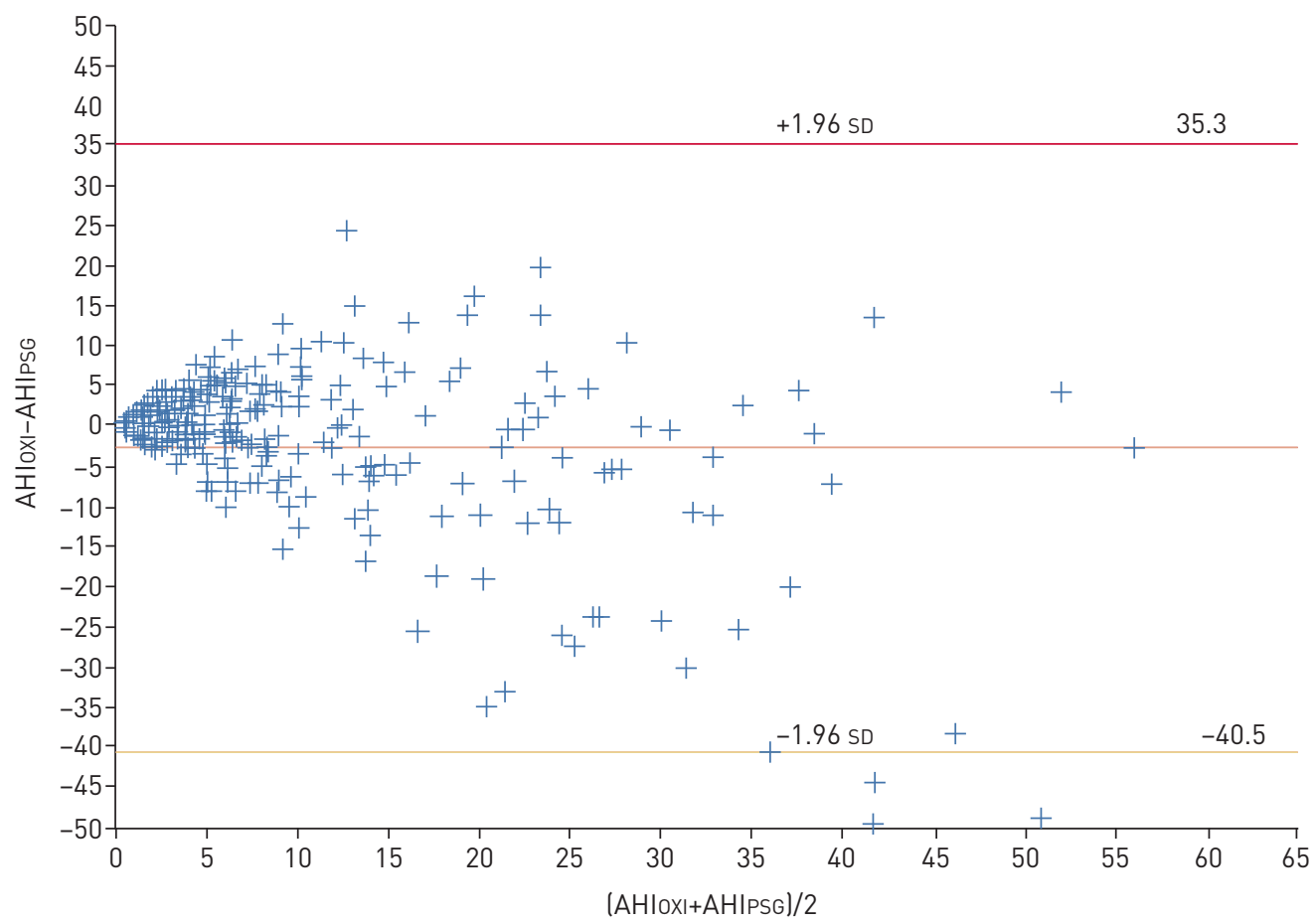

FIGURE 1 Bland-Altman plot comparing polysomnography apnoea/hypopnoea index (AHIPSG) with the estimated AHIoxI from a portable Bluetooth oximeter using a cloud-based algorithm.

Figure 1 displays the Bland-Altman plot comparing the AHIPSG of the subjects with their corresponding and AHIOXI estimation from the oximeter data concurrently acquired during their PSG testing. In addition, a low mean positive difference (slight AHI underestimation by the algorithm) is apparent, with 95\% confidence intervals within (-40.5-35.3), which reflects the dispersion that occurs when AHIPSG values are very high, indicative of extremely severe OSAS. In addition, a high ICC is reached (0.317).

Table 2 and figure 2 show the confusion matrix comparing the classification derived from the AHIPSG with the classification achieved by the cloud-based algorithm based on oximetry alone, i.e. AHIoxI. Accordingly, Cohen's $\kappa$ was 0.339 . In addition, table 2 displays sensitivity, specificity, $\mathrm{LR}^{+}$and $\mathrm{LR}^{-}$for the AHI 1 event $\cdot h^{-1}, 5$ events $\cdot h^{-1}$ and 10 events $\cdot h^{-1}$ cut-offs, derived from the confusion matrix, and figure 3

TABLE 2 Confusion matrix showing the classification agreement of Bluetooth oximeter cloud-based algorithm-calculated apnoea/hypopnoea index (AHI) estimate and the nocturnal polysomnography (PSG)-derived AHI (AHIPSG) in 432 symptomatic habitually snoring Chinese children undergoing overnight polysomnography and concurrent Bluetooth oximetry (OXI) for suspected obstructive sleep apnoea

$\begin{array}{ccc}\text { AHloxl } & \text { Sensitivity Specificity Accuracy } \text { LR }^{+} \\ <1 \text { event } \cdot h^{-1} & 1-5 \text { events } \cdot h^{-1} & 5-10 \text { events } \cdot h^{-1} \geqslant 10 \text { events } \cdot h^{-1} \quad \text { Nocturnal PSG }\end{array}$

\begin{tabular}{lccccc}
\hline AHIPSG & & & & & \\
$<1$ event $\cdot h^{-1}$ & 17 & 66 & 41 & 2 & 89 \\
$1-5$ events $\cdot h^{-1}$ & 14 & 113 & 27 & 18 & 172 \\
$5-10$ events $\cdot h^{-1}$ & 2 & 22 & 13 & 75 & 69 \\
$\geqslant 10$ events $\cdot h^{-1}$ & 0 & 14 & 85 & 99 & 102 \\
$\quad$ Nocturnal OXI & 33 & 215 & 0.339 & & 432 \\
Cohen's & & & &
\end{tabular}

1 event $\cdot h^{-1}$

5 events. $h^{-1}$

10 events $\cdot h^{-1}$

$\begin{array}{cccc}95.3 & 19.1 & 79.6 & 1.18 \\ 77.8 & 80.5 & 79.4 & 3.99 \\ 73.5 & 92.7 & 88.2 & 10.07\end{array}$

$\mathrm{LR}^{+}$: positive likelihood ratio. 

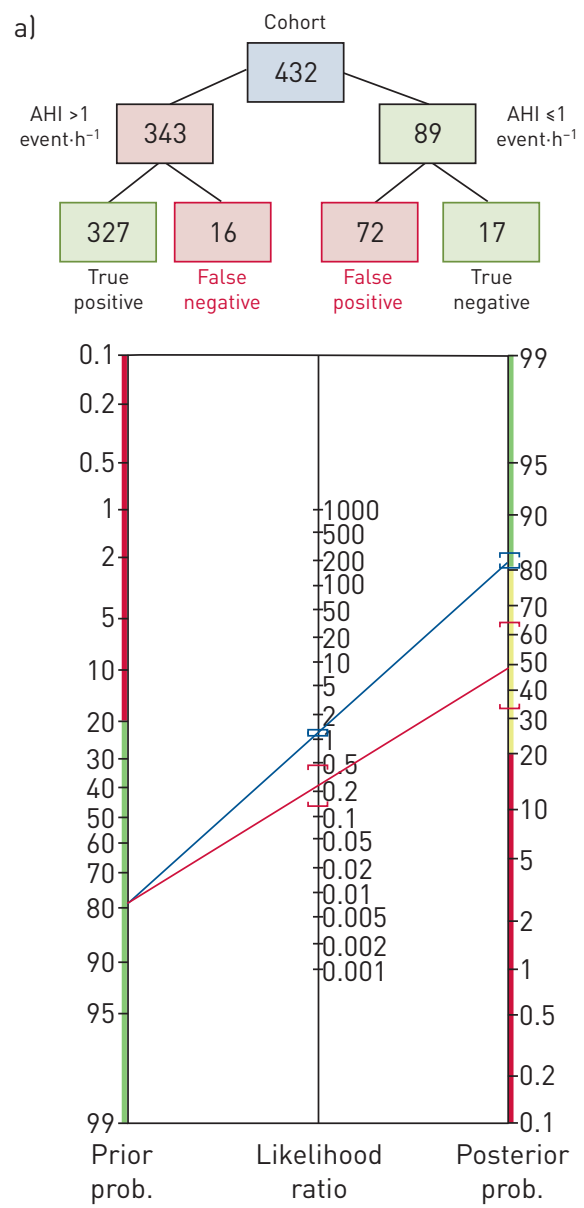
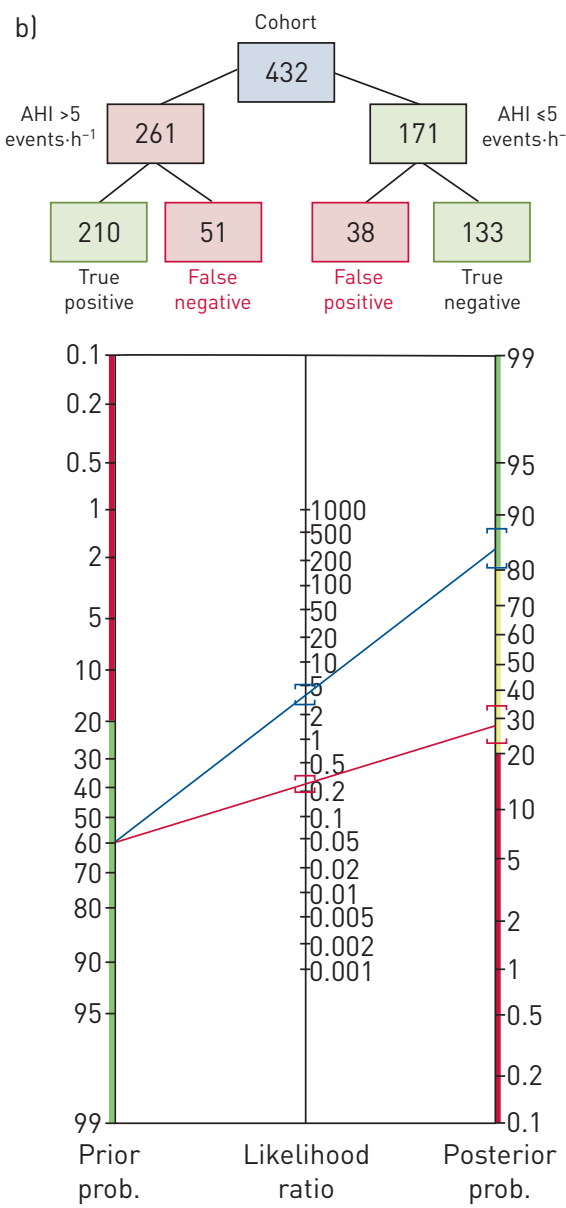
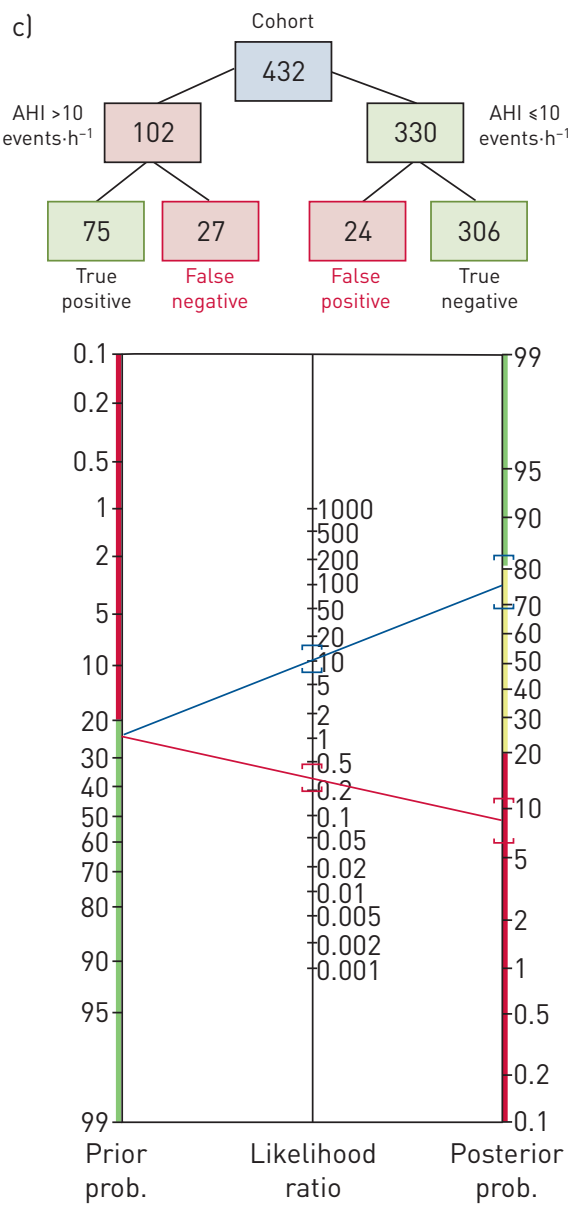

FIGURE 2 Schematic representation of apnoea/hypopnoea index (AHI) oximetry algorithm performance and corresponding likelihood ratios relative to polysomnography-derived AHI when diagnostic cut-off values are set at a) 1 event $\cdot \mathrm{h}^{-1}$, b) 5 events $\cdot \mathrm{h}^{-1}$ or $\mathrm{c}$ ) 10 events $\cdot \mathrm{h}^{-1}$.

displays the receiver operator curves for each of these cut-off values. AHIoxI showed increasing degrees of diagnostic ability as the cut-off increased and became $<90 \%$ specific at AHI cut-off of 10 events. $\mathrm{h}^{-1}$. Accuracies remained $>79 \%$ at all cut-off levels with $\mathrm{LR}^{+}$displaying optimal performance for AHI of $\geqslant 5$ events $\cdot h^{-1}$.

\section{Discussion}

This study shows that a previously developed and validated neural network machine-learning algorithm based on overnight oximetry recordings [29] can be readily and accurately implemented as a readily scalable operator-independent diagnostic tool for the diagnosis of OSAS in symptomatic children referred for evaluation of OSAS.

Before we discuss the clinical implications of current findings, several methodological issues deserve mention. First, only a single commercially available oximeter model was employed for the present study, and displays industry standard accuracy. In this context, awareness of the potential imprecision of the oximeter being selected and employed during implementation of the approach used in the present study is obviously of great importance [34]. Similarly, the potential imprecisions introduced by the finger probe being selected should also be accounted for in materials and supplies selection [35]. However, we should also remark that the heterogeneity of the oximeters and their intrinsic performances was incorporated into the process of derivation and validation of the cloud-based algorithm, and included 13 different paediatric sleep centres around the world using vastly different oximeters and oximeter data-sampling frequencies [29]. Furthermore, the oximeters used in the PSG and the wearable Bluetooth oximeter were different in this study, but achieved concordance in the scored and automatically detected ODI3\%, respectively $\left(r^{2}=0.35\right.$; data not shown). Second, while we attribute the designation of "gold standard" to the PSG, there can be considerable night-to-night variability, particularly in sleep architecture, embedded in the test [36, 38], which may lead to substantial imprecision in the diagnostic decision, particularly at the low end of OSAS severity $[38,39]$. Although not immediately relevant to this study, since both PSG and Bluetooth 
FIGURE 3 Receiver operator curves and corresponding areas under the curve (AUC) when diagnostic apnoea/ hypopnoea index (AHI) cut-off values are set at 1 event $\cdot h^{-1}, 5$ events $\cdot h^{-1}$ or 10 events $\cdot h^{-1}$.

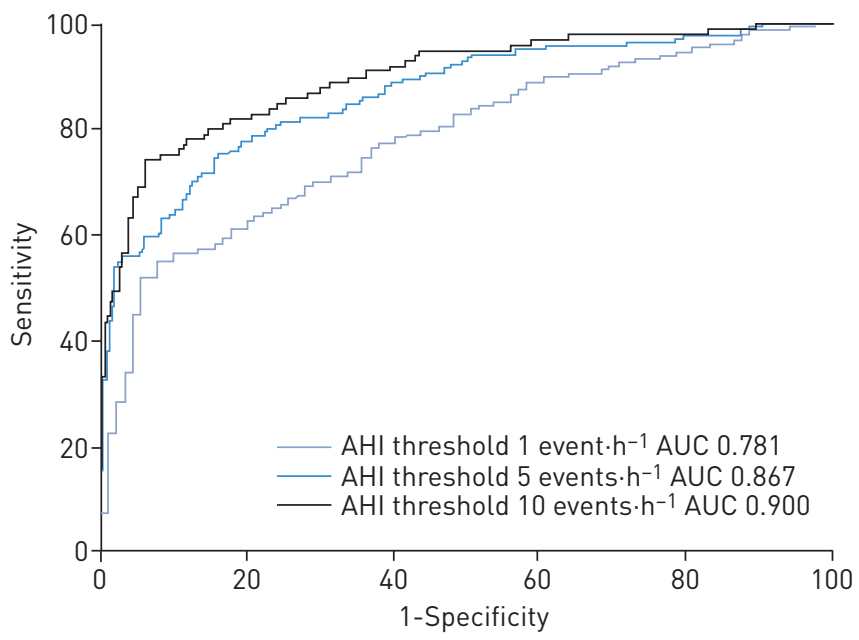

oximetry were implemented concurrently, we should point out that this issue has arisen, albeit inconsistently, in oximetry recordings in children, prompting the recommendation that if a single night yields a negative result in a patient with a high pretest probability, then the addition of two further nights of oximetry recordings should lead to a more reliable clinical decision. [40,41]. Such an approach would be highly feasible with ambulatory oximetry, but impossible with PSG, such that if the accuracies of oximetry-based approaches were virtually indistinguishable from PSG, there would be inherent advantages at using oximetry in this context.

Overall, the accuracy of the portable oximeter cloud-based algorithm dyad was $>79 \%$ for AHI estimates of 1-10 events. $\mathrm{h}^{-1}$, and displayed the anticipated progressive declines in sensitivity with increasing AHI cut-off values to higher specificity at an AHI cut-off of 5 events. $h^{-1}$ (table 2). Thus, and as previously inferred during the process of developing the algorithm, optimal benefits of this automated methodological approach to diagnose OSAS in habitually snoring children [29] is achieved with AHI $\geqslant 5$ events $\cdot h^{-1}$. This cut-off value not only corresponds to a virtually universally agreed-upon equipoise criterion for surgical adenotonsillectomy, but is also associated with an upward inflexion in morbidity risks in children with OSAS [42-44]. However, note that the adoption of the AHI 1 event.h ${ }^{-1}$ cut-off would lead to relatively high rate of false positives, which would then be treated even if their AHIPSG would have been $<1$ event $\cdot h^{-1}$. Conversely, the false negative rate of our approach was small, as illustrated by the fact that using the criterion of AHIPSG $>1$ event $\cdot h^{-1}$, only $4.7 \%$ of false negative cases emerged, from which only $0.6 \%$ of cases would be in the moderate or severe OSAS category. The relatively small proportion of children that would be missed using oximetry-based diagnostics might be further reduced by repeating the oximetry-based test for one or more additional consecutive nights [41], an issue that clearly deserves further exploration in future studies. Furthermore, repeating the test within weeks or months if the child's symptoms persisted would be much more readily achievable than repeating PSG. Thus, the clinical management options and algorithms offered by the PSG, whereby the AHI serves as one of the major parameters guiding clinical intervention, would be indistinguishably afforded by the AHIOXI, albeit at a fraction of the cost and effort.

As alluded previously, the overall scarcity and labour-intensive and financially onerous nature of PSG has prompted the exploration of multiple other suitable diagnostic alternative approaches ranging from questionnaires to ambulatory PSG or to simplified multichannel recordings [45]. However, some such approaches, e.g. respiratory polygraphy, are becoming increasingly accepted as a surrogate diagnostic test in children, despite their reduced accuracy at the low end of the OSAS severity spectrum [10], a limitation that has prompted a lack of endorsement by the AASM [46]. In the present study, our findings clearly show that automated analysis of nocturnal oximetry provides a useful approach to the diagnosis of OSAS among high pretest symptomatic children being referred for evaluation of suspected OSAS. Indeed, and according to the protocol proposed in our previous study which as mentioned above proposed a cut-off of AHI $\geqslant 5$ events $h^{-1}$ [29], OSAS would have been discarded in 38 children, most of them with AHIPSG $<5$ events.h ${ }^{-1}$. In addition, we should remark that several of these children would potentially require treatment anyway, due to concurrent SDB-related morbidity. In addition, 184 subjects would be referred for treatment, with $96.7 \%$ showing mild OSAS. Such an approach would clearly reduce the need for conventional PSG, a finding that is coherent with our previous results [29], whereby $77.8 \%$ children with moderate-to-severe OSAS based on PSG would be identified as such by oximetry coupled to a mobile phone app interfaced with a cloud-based algorithm. Our findings are in close agreement with the 
approach and conclusions from a recent study by PAPADAKIs et al. [47], in which oximetry approaches consisting of ODI $3 \%>3.5$ events $\cdot h^{-1}$ successfully predicted post-surgical intervention outcomes.

In summary, this study provides initial confirmatory demonstration that diagnostic precision can be readily achieved via a portable oximeter linked via a smartphone to a cloud-based automated analytic algorithm. This approach offers not only a valid alternative to standard PSG in the context of childhood OSAS, but also that it is a highly scalable, i.e. incorporates the ability to seamlessly continue to deliver the desired service, in this case oximetry-based diagnostics, in the context of increasing demands in order to meet a user need without requiring additional expert personnel (current rate-limiting factor), and therefore should serve as a remarkably affordable option. Therefore, integrated collection of ambulatory nocturnal oximetry signals and their automated processing by well validated algorithms as the one employed herein, should lead to accurate and widely implementable diagnostic tools for childhood OSAS, thereby enabling timely objective evaluation and treatment with the attendant downstream benefits of reduced morbidity. Inasmuch as the current findings are promising, expanded implementation of the current system to the domiciliary venues and its real life performance in the clinical setting will need to be critically investigated and confirmed.

Conflict of interest: Z. Xu has nothing to disclose. G.C. Gutiérrez-Tobal has nothing to disclose. Y. Wu has nothing to disclose. L. Kheirandish-Gozal acted as a scientific consultant to Serenium Inc., during the conduct of the study. Serenium Inc. graciously provided the oximeter devices, Bluetooth phones and links to algorithm, but was not involved in study design, data acquisition or analysis. X. Ni has nothing to disclose. R. Hornero has nothing to disclose. D. Gozal has nothing to disclose.

Support statement: This work was supported by the Capital Health Research and Development of Special Funding (2018-1-2091); Beijing Municipal Science and Technology Project (Z161100000116050); National Key Research and Development Plan (2017YFC0112502) to X. Ni and Y. Wu; US National Institutes of Health grant HL130984 (L. Kheirandish-Gozal, D. Gozal); and projects DPI2017-84280-R and RTC-2015-3446-1 from "Ministerio de Ciencia, Innovación y Universidades" (Spanish Government) and European Regional Development Fund (FEDER) to G.C. Gutiérrez-Tobal and R. Hornero. Funding information for this article has been deposited with the Crossref Funder Registry.

\section{References}

1 Marcus CL, Brooks LJ, Draper KA, et al. Diagnosis and management of childhood obstructive sleep apnea syndrome. Pediatrics 2012; 130: e714-e755.

2 Tarasiuk A, Simon T, Tal A, et al. Adenotonsillectomy in children with obstructive sleep apnea syndrome reduces health care utilization. Pediatrics 2004; 113: 351-356.

3 Torretta S, Rosazza C, Pace ME, et al. Impact of adenotonsillectomy on pediatric quality of life: review of the literature. Ital J Pediatr 2017; 43: 107.

4 Zhao G, Li Y, Wang X, et al. The predictive value of polysomnography combined with quality of life for treatment decision of children with habitual snoring related to adenotonsillar hypertrophy. Eur Arch Otorhinolaryngol 2018; 275: 1579-1586.

5 Alonso-Âlvarez ML, Canet T, Cubell-Alarco M, et al. Documento de consenso del síndrome de apneas-hipopneas durante el sueño en niños. [Consensus document on sleep apnea-hypopnea syndrome in children]. Arch Bronconeumol 2011; 47: 2-18.

6 Kaditis AG, Alonso Alvarez ML, Boudewyns A, et al. Obstructive sleep disordered breathing in 2- to 18-year-old children: diagnosis and management. Eur Respir J 2016; 47: 69-94.

7 Friedman NR, Perkins JN, McNair B, et al. Current practice patterns for sleep-disordered breathing in children. Laryngoscope 2013; 123: 1055-1058.

8 Lam DJ, Shea SA, Weaver EM, et al. Predictors of obtaining polysomnography among otolaryngologists prior to adenotonsillectomy for childhood sleep-disordered breathing. J Clin Sleep Med 2018; 14: 1361-1367.

9 Kaditis AG, Kheirandish-Gozal L, Gozal D. Algorithm for the diagnosis and management of pediatric OSA: a proposal of two pediatric sleep centers. Sleep Med 2012; 13: 217-227.

10 Alonso-Álvarez ML, Terán-Santos J, Ordax-Carbajo E, et al. Reliability of home respiratory polygraphy for the diagnosis of sleep apnea in children. Chest 2015; 147: 1020-1028.

11 Kaditis AG, Kheirandish-Gozal L, Gozal D. Pediatric OSAS: oximetry can provide answers when polysomnography is not available. Sleep Med Rev 2016; 27: 96-105.

12 Canto Gde L, Pachêco-Pereira C, Aydinoz S, et al. Biomarkers associated with obstructive sleep apnea: a scoping review. Sleep Med Rev 2015; 23: 28-45.

13 Brouillette RT, Morielli A, Leimanis A, et al. Nocturnal pulse oximetry as an abbreviated testing modality for pediatric obstructive sleep apnea. Pediatrics 2000; 105: 405-412.

14 Crespo A, Álvarez D, Kheirandish-Gozal L, et al. Assessment of oximetry-based statistical classifiers as simplified screening tools in the management of childhood obstructive sleep apnea. Sleep Breath 2000; 22: 1063-1073.

15 Vaquerizo-Villar F, Álvarez D, Kheirandish-Gozal L, et al. Utility of bispectrum in the screening of pediatric sleep apnea-hypopnea syndrome using oximetry recordings. Comput Methods Programs Biomed 2018; 156: 141-149.

16 Vaquerizo-Villar F, Alvarez D, Gutierrez-Tobal GC, et al. Usefulness of discrete wavelet transform in the analysis of oximetry signals to assist in childhood sleep apnea-hypopnea syndrome diagnosis. Conf Proc IEEE Eng Med Biol Soc 2017; 2017: 3753-3756.

17 Garde A, Dekhordi P, Petersen CL, et al. Detecting obstructive sleep apnea in children by self-affine visualization of oximetry. Conf Proc IEEE Eng Med Biol Soc 2017; 2017: 3757-3760. 
18 Ma JR, Huang JJ, Chen Q, et al. Value of pulse oximetry watch for diagnosing pediatric obstructive sleep apnea/ hypopnea syndrome. Acta Otolaryngol 2018; 138: 175-179.

19 Álvarez D, Alonso-Álvarez ML, Gutiérrez-Tobal GC, et al. Automated screening of children with obstructive sleep apnea using nocturnal oximetry: an alternative to respiratory polygraphy in unattended settings. J Clin Sleep Med 2017; 13: 693-702.

20 Garde A, Dekhordi P, Ansermino JM, et al. Identifying individual sleep apnea/hypoapnea epochs using smartphone-based pulse oximetry. Conf Proc IEEE Eng Med Biol Soc 2016; 2016: 3195-3198.

21 Pavone M, Ullmann N, Verrillo E, et al. At-home pulse oximetry in children undergoing adenotonsillectomy for obstructive sleep apnea. Eur J Pediatr 2017; 176: 493-499.

22 Nixon GM, Davey MJ, Weichard AJ, et al. Oximetry for suspected obstructive sleep apnea - does removal of awake data affect the result? Pediatr Pulmonol 2016; 51: 1409-1413.

23 Garde A, Dehkordi P, Wensley D, et al. Pulse oximetry recorded from the Phone Oximeter for detection of obstructive sleep apnea events with and without oxygen desaturation in children. Conf Proc IEEE Eng Med Biol Soc 2015; 2015: 7692-7695.

24 Gutierrez-Tobal GC, Kheirandish-Gozal L, Alvarez D, et al. Analysis and classification of oximetry recordings to predict obstructive sleep apnea severity in children. Conf Proc IEEE Eng Med Biol Soc 2015; 2015: 4540-4543.

25 Alvarez D, Kheirandish-Gozal L, Gutierrez-Tobal GC, et al. Automated analysis of nocturnal oximetry as screening tool for childhood obstructive sleep apnea-hypopnea syndrome. Conf Proc IEEE Eng Med Biol Soc 2015; 2015: 2800-2803.

26 Villa MP, Pietropaoli N, Supino MC, et al. Diagnosis of pediatric obstructive sleep apnea syndrome in settings with limited resources. JAMA Otolaryngol Head Neck Surg 2015; 141: 990-996.

27 Van Eyck A, Lambrechts C, Vanheeswijck L, et al. The role of nocturnal pulse oximetry in the screening for obstructive sleep apnea in obese children and adolescents. Sleep Med 2015; 16: 1409-1412.

28 Gozal D, Kheirandish-Gozal L, Kaditis AG. Home sleep testing for the diagnosis of pediatric obstructive sleep apnea: the times they are a changing...! Curr Opin Pulm Med 2015; 21: 563-568.

29 Hornero R, Kheirandish-Gozal L, Gutiérrez-Tobal GC, et al. Nocturnal oximetry-based evaluation of habitually snoring children. Am J Respir Crit Care Med 2017; 196: 1591-1598.

30 Zong Y, Xie R, Deng N, et al. Secular trends in overweight and obesity among urban children and adolescents, 2003-2012: a serial cross-sectional study in Guangzhou, China. Sci Rep 2017; 7: 12042.

31 Berry RB, Budhiraja R, Gottlieb DJ, et al. Rules for scoring respiratory events in sleep: update of the 2007 AASM Manual for the Scoring of Sleep and Associated Events. Deliberations of the Sleep Apnea Definitions Task Force of the American Academy of Sleep Medicine. J Clin Sleep Med 2012; 8: 597-619.

32 Magalang UJ, Dmochowski J, Veeramachaneni S, et al. Prediction of the apnea-hypopnea index from overnight pulse oximetry. Chest 2003; 124: 1694-1701.

33 Bland JM, Altman DG. Statistical methods for assessing agreement between two methods of clinical measurement. Lancet 1986; 1: 307-310.

34 Lipnick MS, Feiner JR, Au P, et al. The accuracy of 6 inexpensive pulse oximeters not cleared by the Food and Drug Administration: the possible global public health implications. Anesth Analg 2016; 123: 338-345.

35 van Oostrom JH, Melker RJ. Comparative testing of pulse oximeter probes. Anesth Analg 2004; 98: 1354-1358.

36 Katz ES, Greene MG, Carson KA, et al. Night-to-night variability of polysomnography in children with suspected obstructive sleep apnea. J Pediatr 2002; 140: 589-594.

37 Scholle S, Scholle HC, Kemper A, et al. First night effect in children and adolescents undergoing polysomnography for sleep-disordered breathing. Clin Neurophysiol 2003; 114: 2138-2145.

38 Li AM, Wing YK, Cheung A, et al. Is a 2-night polysomnographic study necessary in childhood sleep-related disordered breathing? Chest 2004; 126: 1467-1472.

39 Ahmadi N, Shapiro GK, Chung SA, et al. Clinical diagnosis of sleep apnea based on single night of polysomnography $v s$. two nights of polysomnography. Sleep Breath 2009; 13: 221-226.

40 Pavone M, Cutrera R, Verrillo E, et al. Night-to-night consistency of at-home nocturnal pulse oximetry testing for obstructive sleep apnea in children. Pediatr Pulmonol 2013; 48: 754-760.

41 Burke RM, Maxwell B, Hunter C, et al. Night-to-night variation of pulse oximetry in children with sleep-disordered breathing. Arch Dis Child 2016; 101: 1095-1099.

42 Bhattacharjee R, Kim J, Alotaibi WH, et al. Endothelial dysfunction in children without hypertension: potential contributions of obesity and obstructive sleep apnea. Chest 2012; 141: 682-691.

43 Gozal D, Kheirandish-Gozal L, Bhattacharjee R, et al. Neurocognitive and endothelial dysfunction in children with obstructive sleep apnea. Pediatrics 2010; 126: e1161-e1167.

44 Hunter SJ, Gozal D, Smith DL, et al. Effect of sleep-disordered breathing severity on cognitive performance measures in a large community cohort of young school-aged children. Am J Respir Crit Care Med 2016; 194: 739-747.

45 Tan HL, Kheirandish-Gozal L, Gozal D. Pediatric home sleep apnea testing: slowly getting there! Chest 2015; 148: 1382-1395.

46 Kirk V, Baughn J, D'Andrea L, et al. American Academy of Sleep Medicine position paper for the use of a home sleep apnea test for the diagnosis of OSA in children. J Clin Sleep Med 2017; 13: 1199-1203.

47 Papadakis CE, Chaidas K, Chimona TS, et al. Use of oximetry to determine need for adenotonsillectomy for sleep-disordered breathing. Pediatrics 2018; 142: e20173382. 